\title{
The TESLA Time Projection Chamber
}

\author{
N. Ghodbane ${ }^{\mathrm{a} *}$ \\ (for the ECFA-DESY TPC study for a Linear Electron-Positron Collider)
}

${ }^{a}$ Deutsches Elektronen Synchrotron,

Notkestraße 85, 22607 Hamburg, Deutschland

A large Time Projection Chamber is proposed as part of the tracking system for a detector at the TESLA electron positron linear collider. Different ongoing R\&D studies are reviewed, stressing progress made on a new type readout technique based on Micro-Pattern Gas Detectors.

\section{Introduction}

TESLA (TeV-Energy Superconducting Linear Accelerator) is an $e^{+} e^{-}$linear Collider designed to operate in the energy range beyond the mass of the $\mathrm{Z}^{0}$ and up to the $\mathrm{TeV}$ region. If approved, TESLA would allow a wide physics program, focusing among other things on precision tests of the established Standard Model, the Higgs mechanisms and the discovery of new particles.

To optimally exploit the physics potential of TESLA, the detector has to satisfy some stringent requirements. It has to have a very good track momentum resolution to measure the $\mathrm{Z}^{0}$ recoil mass, an excellent vertex resolution for heavy flavour identification, a high resolution in the reconstruction of electromagnetic and hadronic showers in the calorimeter, and has to be as hermetic as possible. The general concept of a detector fulfilling these criteria, discussed within the ECFA-DESY study, is described in details in $[1,2]$.

In this contribution, we describe the current design of the Time Projection Chamber (TPC) and the different ongoing studies concerning the readout technologies.

\section{The TESLA central Tracker}

A large TPC is proposed as the main inner tracker at TESLA. The actual design, taking into

${ }^{*}$ Speaker at the $8^{\text {th }}$ Topical Seminar on Innovative Particle and Radiation Detectors Siena, 21-24 October 2002 account the different performance goals, is described in details in the TESLA TDR [2].

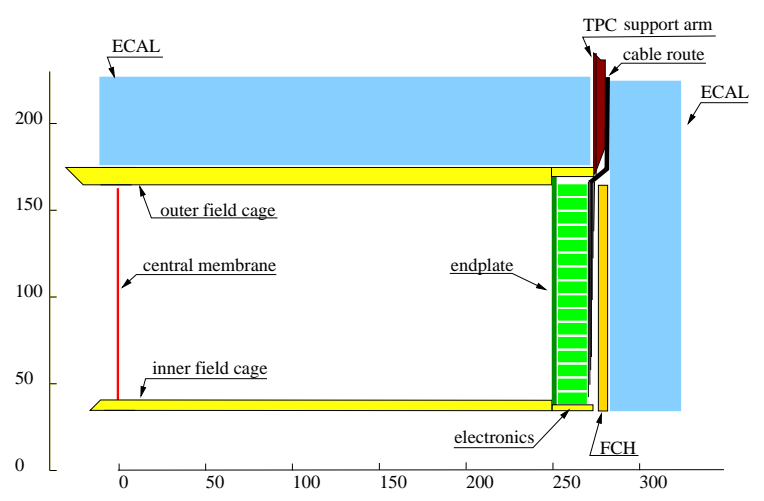

Figure 1. General layout of one quarter of the central tracking.

The TPC, as shown schematically in figure 1, is of cylindrical design with an inner radius of about $32 \mathrm{~cm}$, an outer radius of about $170 \mathrm{~cm}$ and an overall length in the beam direction of about $2 \times 273 \mathrm{~cm}$. The internal radius is determined by the size of the mask system, whereas the external radius is constrained by the fact that the calorimeter is inside the coil. These large dimensions allow the tracks to be measured with about $200(r, \phi, z)$ space points, so that the 
global tracking efficiency remains close to $100 \%$ in the overall volume and a momentum resolution $\delta\left(1 / p_{t}\right)<1.5 \times 10^{-4} / \mathrm{GeV}$ in the central region can be achieved. In addition the TPC should measure the specific energy loss of particles thus aiding in the identification of charged particles. With Ar-based gas mixtures, operating at atmospheric pressure, it is expected that a resolution of $\delta(\log (\mathrm{dE} / \mathrm{dx})) \leq 4.5 \%$ can be reached, enough, to provide a separation between pions and kaons of better than two sigma for momenta between 2 and $20 \mathrm{GeV}$.

Operating a TPC in the environment at TESLA presents a number of challenges. Presently no dedicated hardware trigger is forseen within a bunch train, so that all detectors should be active throughout one full train about $1 \mathrm{~ms}$. One readout cycle of the TPC will integrate over approximatively 150 bunch crossings at TESLA, and many readout cycles will follow continuously until a train has passed. This makes it important that the readout is granular enough to resolve the expected number of hits. In addition means have to be found to avoid the need for gating the TPC to remove the buildup of positive ions: during one bunch trains ions will travel significantly into the TPC drift volume, potentially distorting the drift field.

A possible solution to handle both the high granularity and to suppress the ions drift into the drift volume is the use of Micro-Pattern Gas Detectors as readout devices. Possible candidates are at the moment MicroMEGAS chambers [3] or Gas Electron Multipliers (GEM) foils [4].

Micro-Pattern Gas Chambers, due to their intrinsically smaller distances between amplification elements compared to the traditional wire chambers, offer the promise to significantly reduce systematic distortions in the electron drift close to the amplification region due to the so-called $\overrightarrow{\mathrm{E}} \times \overrightarrow{\mathrm{B}}$ effect.

\section{Ongoing Studies}

A challenging Research and Development program is underway to meet the current design goals for a TPC at a linear collider like TESLA. Institutes from a number of different countries are participating (Aachen, Carleton, DESY, IPN Orsay, Karlsruhe, Krakow, LAL, LBNL, MIT, MPI Munich, NIKHEF, Novosibirsk, Rostock, Saclay and Victoria). The main focus of the work is at the moment on the development of a valid amplification and ion-suppression scheme (see reference [5]). Over the next years, efforts to develop a compact readout scheme and to understand the design of the mechanical structure of such a large TPC are planned. In the following sections we present the different first results for these different key issues.

\subsection{Gas Amplification Systems}

Several studies focus on the TPC readout using Micro-Pattern Gas Detectors instead of the wire used conventionally to produce the gas amplification that is necessary to read the TPC signal. Gas Electron Multiplier (GEM) and MicroMEGAS, sketched in figure 2, are two attractive candidates for the readout planes.

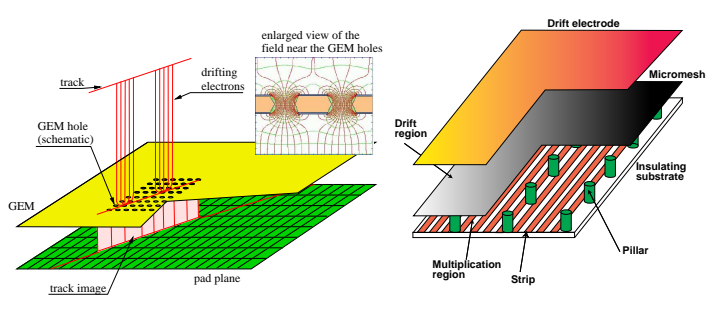

Figure 2. Sketch of the GEM (left) and MicroMEGAS.

A GEM foil is a sandwich consisting of thin conductive layers of copper separated by a thin polymer film of capton. The foil is perforated with small holes arranged in a hexagonal lattice. When operating in the detector, a potential applied to the two conductive layers generates a strong electric field, typically $80 \mathrm{kV} / \mathrm{cm}$, in the small holes. The electric field lines guide charged particles through the GEM holes, with minimal losses due to collisions with the foil. Electrons are accelerated in the strong field lines within the GEM foil, producing secondary ionizations and charge 
multiplication which are then transfered by the electric field to the readout pads where they are collected. A unique property of the GEMs is their capability to operate in cascade, i.e. in a multiGEM structure, offering several advantages like a higher gain and an effective ion feedback suppression. Most of the groups working with GEM readout use GEM towers with two or more GEMs. An attractive alternative technology to GEM is that of MicroMEGAS. An uniform high-field, typically $30 \mathrm{kV} / \mathrm{cm}$, is produced between a thin metallic mesh, few microns thick, stretched at a distance of 50-100 $\mu \mathrm{m}$ above the readout pad plane and held by insulating pillars. On top of the mesh, the electrons are transferred to the amplification gap, where they are multiplied in an avalanche process, resulting in a large number of electron-ion pairs. A MicroMEGAS has similar advantages to a GEM as far as simplicity of construction, low cost and efficiency of ion-feedback suppression are concerned. It has an excellent potential for $\mathrm{dE} / \mathrm{dx}$ resolution due to the fact that the gain is nearly independent of the gap thickness. Moreover it is robust and can be built at least in parts using commercially available components.

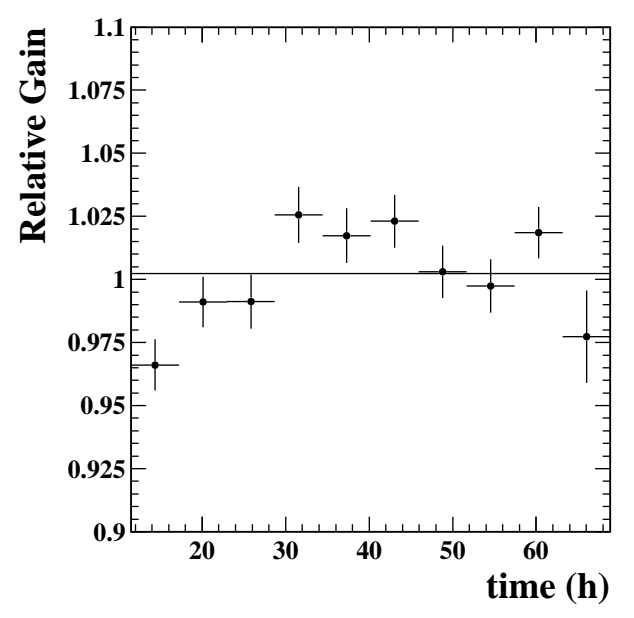

Figure 3. Gain uniformity without atmospheric corrections of a two-GEM structure using the TESLA TDR Gas.
R\&D studies focus among other things on the gain stability and homogeneity to reach a $\mathrm{dE} / \mathrm{dx}$ measurement with $5 \%$ precision. This is illustrated in figure 3 which shows the gain uniformity with time using a two-GEM tower structure.

\subsection{Ion Feedback Suppression}

A crucial issue at TESLA is to prevent the ions produced during the amplification to return to the TPC drift volume and cause field distortions. The use of GEMs or MicroMEGAS promises to reduce the ion feedback. The degree of reduction depends strongly on the exact choice of operational parameters and the Micro-Pattern Gas Detector used. Several ongoing studies, using GEMs and MicroMEGAS, demonstrated a suppression to the level of $10^{-2}$ (figure 4). However, a level of suppression to a level of $10^{-4}$ is needed to ensure that the positive-ion effects are at an acceptable level. In case this goal cannot be reached and to guarantee a stable and robust chamber operation, the insertion of a gating plane, gated in between bunch trains, might be needed in order to minimize the effects of ion feedback.

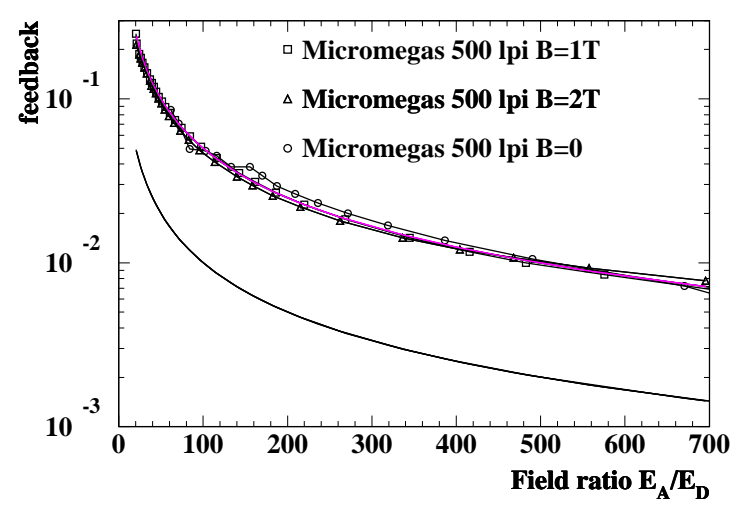

Figure 4. Ion feedback suppression using MicroMEGAS for different electric and magnetic field configurations. 


\subsection{Simulations Studies}

To optimally exploit GEMs and MicroMEGAS and in addition to the experimental program, extensive simulation studies are being performed. These concern mainly the study of the charge transfer mechanism in GEM or MicroMEGAS structures. These numerical simulations are of crucial importance, since they determine the optimal parameter choice needed to reach the different TESLA TPC milestones.

Figure 5 shows, as the validation of the simulation of a GEM hole, the comparison between measurement and simulation of the evolution of the primary and secondary extraction efficiencies respectively defined as the fraction of ions extracted from the GEM holes into the drift volume per number of ions produced in the holes and the fraction of ions extracted from the GEM hole into the drift volume per number of ions which have been collected in the GEM hole.

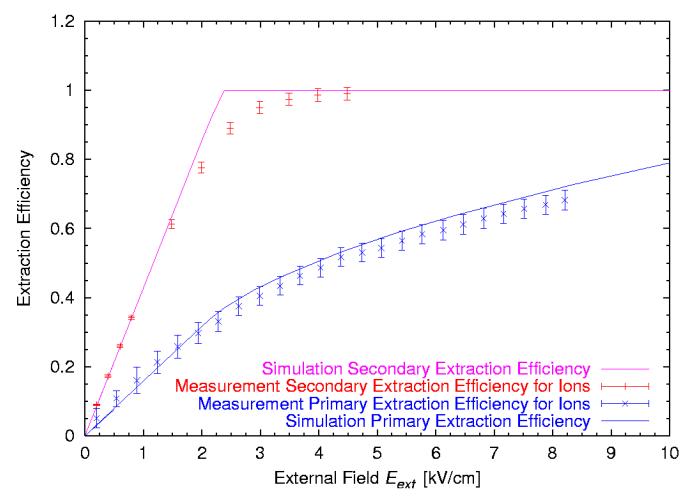

Figure 5. Comparison of data and simulation for the ion extraction efficiency from a GEM hole.

\subsection{Resolution and Pad Geometry Studies}

The charge produced during the avalanche in the GEMs is collected by readout pads located typically a few $\mathrm{mm}$ behind the last GEM. Since the number of pads is limited, the distance between pad centers is large compared to the size of the electron avalanche cloud (typically a few to several $100 \mu \mathrm{m}$ ). The charge from a track after amplification in the GEMs is therefore sometimes collected on a single pad. This effect is even more important with magnetic field at TESLA since the transverse diffusion is then smaller. The major consequence will be that the centre of gravity method cannot be applied anymore for position calculation. Ongoing simulation studies investigate more sophisticated pad geometries, like chevron-shaped pads which may lead to a better point resolution if the charge sharing between neighboring pads is more important.

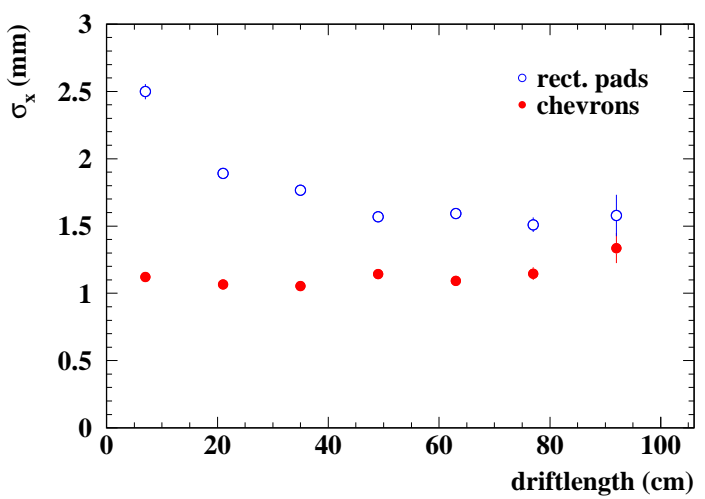

Figure 6. Point resolution as a function of the drift length for two pad geometries, chevrons and rectangular pads.

Figure 6 shows the measured point resolution $\sigma_{x}$ for rectangular and chevron shaped pad with a size of $14 \times 14 \mathrm{~mm}^{2}$ and for different drift lengths using GEMs and without magnetic field. For small drift distances the point resolution with chevrons is better than with rectangles. This effect of the pad geometry gets smaller with increasing drift distances since the influence of the diffusion of the electron cloud gets larger. The improvement is clearly visible but simulation studies concerning mainly the pad response function description, the optimal induction gap between the last GEM and the pad plane, are still required 
for a better description of the pad charge sharing mechanism.

Another approach to make the electron charge cloud broader, and get then a better point resolution, uses a resistive anode foil placed a few ten of $\mu \mathrm{m}$ between the readout plane and the last GEM. This work is illustrated by the plot on figure 7 showing the signal detected with a size of about $2 \mathrm{~mm}$ for an initial charge cluster size of $1 \mathrm{~mm}$.

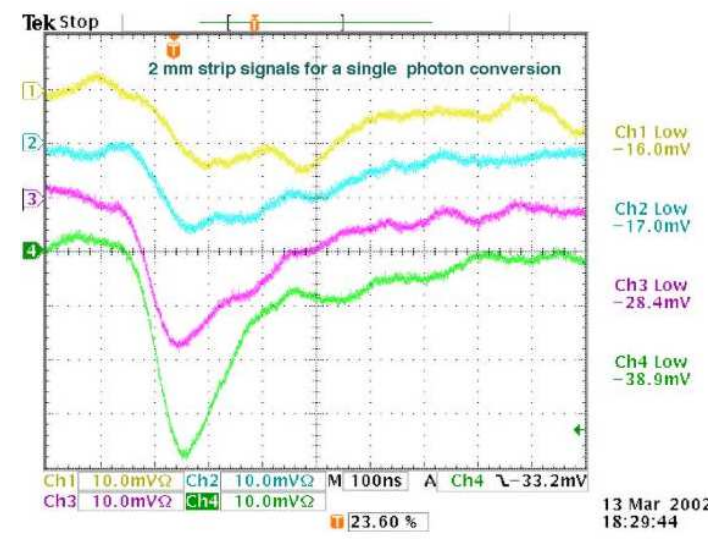

Figure 7. A TPC event using a resistive anode GEM.

\subsection{Induced Pulses in a GEM}

The possibility to detect induced pulses in a GEM setup is also being studied. During the buildup of charges in the GEM hole a small induced pulse is expected in the pad opposite to this hole. This signal is also visible in the neighbor pads and can then be used to reconstruct the position of the avalanche and have a resolution significantly better. The price to pay for this improvement is that the readout electronics has to be able to detect the small pulses and that a very fast electronics is needed.

\section{Conclusion and Outlook}

Several institutes have started a joint R\&D effort with the goal of developing the technologies needed for a large TPC at the next generation of linear colliders. The main challenge is to design a continuous-tracking, high performance TPC which should be substantially more performant than any existing TPC. Important progress has been made to better understand the different aspects of the TPC. Particular emphasis is placed on the amplification, the ion-suppression scheme and the large scale-integration electronics. While first results concerning these different points look very promising, some open questions like the readout behavior in a strong magnetic field, or the results with large TPC prototypes, still remain unanswered and need more investigations in the near future.

\section{Acknowledgments}

I wish to thank all the members of the ECFADESY TPC study for providing material for this contribution. I'm grateful to Ties Behnke for reading this manuscript.

\section{REFERENCES}

1. The TESLA detector; J.Mnich, proceeding for the $8^{t h}$ Topical Seminar on Innovative Particle and Radiation Detectors Siena, 2124 October 2002.

2. TESLA Technical Design Report, Part IV: A Detector for TESLA, editors: T.Behnke, S. Bertolucci, R.-D. Heuer, R. Settles, 2001.

3. Y. Giomataris et al., Nucl. Instrum. Meth. A 376 (1996) 29.

4. F. Sauli,Nucl. Instrum. Meth. A 386 (1997) 531.

5. A TPC for a Future Linear Collider, the LC TPC Group, LC-DET-2002-008, http://www.desy.de/lcnotes. 